\title{
Survey of Fluctuating Fetal Body Weights in Sprague-Dawley Rats
}

\author{
Yoshiki BAN, Tetsuro KOMATSU, Toshio NAKATSUKA, \\ Hiroyoshi MATSUMOTO, Motoshi KONDO, \\ and Takaaki FUJI**
Development Research Laboratories, Banyu Pharmaceutical Co., Ltd., 810 Nishijo, Menuma-machi, Osato-gun, Saitama 360-02 and ${ }^{*}$ Research and Development Headquarters, Banyu Pharmaceutical Co., Ltd., 2-9-3 Shimomeguro, Meguro-ku, Tokyo 153, Japan

(Received 22 April 1993/Accepted 3 September 1993)

\begin{abstract}
During the past 9 years of reproductive and developmental toxicity studies in SpragueDawley rat, we noticed that the average fetal body weight on day 20 of gestation in 81 control groups fluctuated significantly from study to study. The other parameters such as number of implants, resorption rats and number of live fetuses were relatively stable. With information cooperatively given by the animal supplier, we could pin down the possibility of the fluctuation. The animal supplier introduced Sprague-Dawley rats into Japan from the U. S. twice in the past. The documents we analyzed indicated that the fluctuation in fetal body weight was caused by different two colonies which were introduced into Japan at the different times. - KEY WORDS : fetal-weight, rats, Sprague-Dawley
\end{abstract}

In order to accurately evaluate study results, it is important to accumulate control data and to compare them with each study result particularly in toxicology studies. At the same time, quality of the control data must be closely monitored to make the comparison relevant.

We have been using a strain of SpragueDawley rats for developmental and reproductive toxicity studies since 1983 . In the studies pregnant rats were cesarean-sectioned in the morning on gestational day 20 (plug day=day $0)$ and their reproductive parameters were examined. During the course of studies, we noticed that the average fetal body weights in the control group were fluctuating from study to study. This report discusses the cause of the fluctuation of fetal body weights.

Table 1 shows reproductive status data (litter mean) obtained from 81 control groups during the past 9 years from March 1983 to February 1992. The average number of pregnant females in a group was 19.2 with the minimum and maximum numbers 6 and 95, respectively. There was fluctuation in average fetal body weights with the lowest and highest values of 2.88 and $3.77 \mathrm{~g}$, respectively. The other reproductive parameters of average number of implants, resorption rates and number of live fetuses were relatively stable.

First, we tried to find a clue to the variation, relating the results with any other intralitter and-study result or environmental factor documented in each study without any positive findings. At the same time, we started to use only animals from one (B) of two breeding centers, as the supplier had two breeding centers (A and B) and B seemed to have supplied animals with more stable fetal weights.

As shown in Fig. 1, the animal supplier started the business with a breeding center (A) and later in 1985 added a new breeding center (B) to expand the business. Since we did not specify the center to obtain the animals, we had been supplied with animals either from A or $\mathrm{B}$ in random fashion. We are also informed that the animals had been introduced into Japan from the U.S. twice at different times, once in 
Table 1. Historical control data of reproductive status for the past 9 years

\begin{tabular}{|c|c|c|c|c|c|c|c|c|c|c|c|}
\hline $\begin{array}{l}\text { C-section } \\
\text { date }(\mathrm{m} / \mathrm{y})\end{array}$ & $\begin{array}{l}\text { No. preg. } \\
\text { females }\end{array}$ & $\begin{array}{c}\text { No. } \\
\text { implants }\end{array}$ & $\begin{array}{l}\text { Resorp. } \\
\text { rate }(\%)\end{array}$ & $\begin{array}{l}\text { No. live } \\
\text { fetuses }\end{array}$ & $\begin{array}{c}\text { Fetal } \\
\text { BW (g) }\end{array}$ & $\begin{array}{l}\text { C-section } \\
\text { date }(\mathrm{m} / \mathrm{y})\end{array}$ & $\begin{array}{l}\text { No. preg. } \\
\text { females }\end{array}$ & $\begin{array}{c}\text { No. } \\
\text { implants }\end{array}$ & $\begin{array}{l}\text { Resorp. } \\
\text { rate }(\%)\end{array}$ & $\begin{array}{l}\text { No. live } \\
\text { fetuses }\end{array}$ & $\begin{array}{c}\text { Fetal } \\
\text { BW (g) }\end{array}$ \\
\hline $3 /{ }^{\prime} 83$ & 25 & 13.2 & 6.0 & 12.4 & 3.53 & $8 /{ }^{\prime} 87$ & 20 & 16.0 & 4.1 & 15.4 & 3.16 \\
\hline 3 & 23 & 15.1 & 5.1 & 14.7 & 3.46 & 9 & 21 & 16.0 & 6.1 & 15.0 & 3.28 \\
\hline 3 & 22 & 12.0 & 5.2 & 11.5 & 3.50 & 9 & 11 & 15.4 & 3.6 & 14.8 & 3.17 \\
\hline 3 & 25 & 14.6 & 6.3 & 13.8 & 3.43 & 11 & 25 & 15.8 & 7.8 & 14.7 & 3.33 \\
\hline 3 & 11 & 14.2 & 4.9 & 13.4 & 3.48 & 11 & 11 & 15.1 & 7.9 & 14.2 & 3.13 \\
\hline 6 & 12 & 15.4 & 5.3 & 14.5 & 3.16 & 12 & 13 & 14.8 & 5.5 & 14.0 & 3.20 \\
\hline 11 & 12 & 15.7 & 7.3 & 14.6 & 3.11 & 12 & 19 & 16.6 & 5.3 & 15.7 & 3.21 \\
\hline 11 & 15 & 14.3 & 10.4 & 12.9 & 2.88 & $1 / ' 88$ & 20 & 16.5 & 7.2 & 15.3 & 3.19 \\
\hline 12 & 31 & 12.6 & 6.0 & 11.9 & 3.04 & 1 & 25 & 16.3 & 6.4 & 15.3 & 3.37 \\
\hline $1 / ' 84$ & 22 & 14.2 & 7.1 & 13.1 & 3.24 & 3 & 13 & 15.2 & 3.1 & 14.7 & 3.13 \\
\hline 1 & 18 & 12.5 & 4.9 & 11.9 & 3.24 & 5 & 11 & 15.1 & 8.3 & 13.9 & 3.26 \\
\hline 1 & 26 & 13.9 & 5.9 & 13.0 & 3.22 & 9. & 10 & 15.7 & 1.3 & 15.5 & 3.36 \\
\hline 1 & 15 & 14.5 & 5.1 & 13.7 & 3.30 & 9 & 11 & 14.5 & 6.3 & 13.8 & 3.20 \\
\hline 1 & 19 & 13.5 & 4.5 & 12.8 & 3.31 & 10 & 10 & 15.1 & 4.9 & 14.4 & 3.22 \\
\hline 1 & 15 & 13.8 & 5.9 & 13.0 & 3.32 & 10 & 95 & 14.4 & 4.5 & 13.7 & 3.60 \\
\hline 2 & 11 & 14.2 & 5.2 & 13.4 & 3.22 & 11 & 16 & 14.8 & 4.8 & 14.0 & 3.25 \\
\hline 4 & 14 & 15.0 & 6.2 & 14.1 & 3.29 & 2'’'89 & 25 & 14.7 & 6.5 & 14.3 & 3.60 \\
\hline 4 & 39 & 13.1 & 3.6 & 12.6 & 3.20 & 3 & 12 & 15.6 & 3.2 & 15.0 & 3.64 \\
\hline 5 & 20 & 12.4 & 4.8 & 11.6 & 3.29 & 6 & 24 & 15.8 & 2.6 & 15.4 & 3.63 \\
\hline 6 & 13 & 13.2 & 3.9 & 12.6 & 3.07 & 6 & 11 & 14.6 & 6.5 & 13.5 & 3.63 \\
\hline 8 & 18 & 14.6 & 6.4 & 13.7 & 3.10 & 7 & 23 & 14.6 & 3.0 & 14.2 & 3.67 \\
\hline 10 & 22 & 15.4 & 7.8 & 14.3 & 3.33 & 9 & 25 & 14.0 & 4.3 & 13.4 & 3.76 \\
\hline 10 & 24 & 15.4 & 6.3 & 14.5 & 3.23 & 9 & 11 & 13.5 & 5.0 & 12.9 & 3.65 \\
\hline 12 & 11 & 13.3 & 10.1 & 12.4 & 3.51 & 10 & 20 & 14.6 & 3.5 & 14.0 & 3.59 \\
\hline $1 / ' 85$ & 14 & 15.9 & 3.5 & 15.4 & 3.53 & $3 / ' 90$ & 21 & 15.3 & 5.2 & 14.5 & 3.66 \\
\hline 1 & 12 & 15.8 & 5.2 & 15.0 & 3.59 & 4 & 24 & 15.9 & 6.2 & 14.9 & 3.60 \\
\hline 2 & 21 & 14.8 & 6.4 & 13.8 & 3.58 & 4 & 23 & 13.9 & 6.9 & 13.0 & 3.70 \\
\hline 4 & 24 & 14.8 & 3.2 & 14.3 & 3.58 & 8 & 24 & 16.2 & 5.2 & 15.3 & 3.53 \\
\hline 7 & 24 & 16.0 & 5.9 & 15.0 & 3.60 & 9 & 9 & 15.7 & 4.2 & 15.1 & 3.77 \\
\hline 9 & 12 & 15.4 & 3.5 & 14.8 & 3.63 & 11 & 11 & 15.8 & 3.0 & 15.4 & 3.65 \\
\hline 9 & 15 & 15.2 & 6.4 & 14.3 & 3.49 & $1 / ' 91$ & 23 & 15.0 & 5.6 & 14.2 & 3.61 \\
\hline 10 & 11 & 15.4 & 4.4 & 14.6 & 3.20 & 1 & 25 & 16.4 & 4.1 & 15.7 & 3.54 \\
\hline 11 & 24 & 15.0 & 3.7 & 14.4 & 3.31 & 6 & 8 & 16.0 & 8.7 & 14.8 & 3.30 \\
\hline $1 / ' 86$ & 24 & 15.8 & 5.2 & 15.0 & 3.15 & 6 & 24 & 16.1 & 5.5 & 15.2 & 3.77 \\
\hline 1 & 11 & 14.8 & 5.8 & 14.1 & 3.04 & 10 & 23 & 13.1 & 7.7 & 12.0 & 3.65 \\
\hline 5 & 20 & 14.9 & 3.7 & 14.4 & 3.05 & 11 & 23 & 14.3 & 6.3 & 13.4 & 3.64 \\
\hline 6 & 15 & 13.7 & 4.5 & 13.1 & 3.12 & 12 & 30 & 15.0 & 4.9 & 14.3 & 3.67 \\
\hline 9 & 12 & 16.5 & 7.6 & 15.2 & 3.26 & $1 /{ }^{\prime} 92$ & 23 & 15.8 & 1.9 & 15.4 & 3.68 \\
\hline 11 & 25 & 16.3 & 6.3 & 15.2 & 3.25 & 2 & 23 & 14.8 & 2.1 & 14.5 & 3.64 \\
\hline 12 & 13 & 12.6 & 9.4 & 11.7 & 3.14 & 2 & 6 & 13.3 & 2.9 & 12.8 & 3.52 \\
\hline $5 / ' 87$ & 21 & 15.9 & 9.8 & 14.4 & 3.28 & & & & & & \\
\hline
\end{tabular}

1970's (Colony 1) and the other in early 1980's (Colony 2). According to the document those animals of different colonies were never crossbred. Then, we classified the average weights into three groups, Colony 1, Colony 2 and Colony unidentified as shown in Fig. 2. There was apparent difference in the fetal weight between Colonies 1 and 2. The statistical comparison between the weights of the two colonies clearly showed a significant difference $(\mathrm{P} \leq$
$0.05)$ with the averages of $3.61 \mathrm{~g}$ for Colony 1 and $3.21 \mathrm{~g}$ for Colony 2 , whereas there were no significant $(\mathrm{P}>0.05)$ differences in number of implants, resorption rate and number of live fetuses between Colonies 1 and 2, as shown in Table 2 .

The result of our survey indicated that the fluctuation in fetal body weight was caused by different two colonies which were introduced into Japan at the different time. 


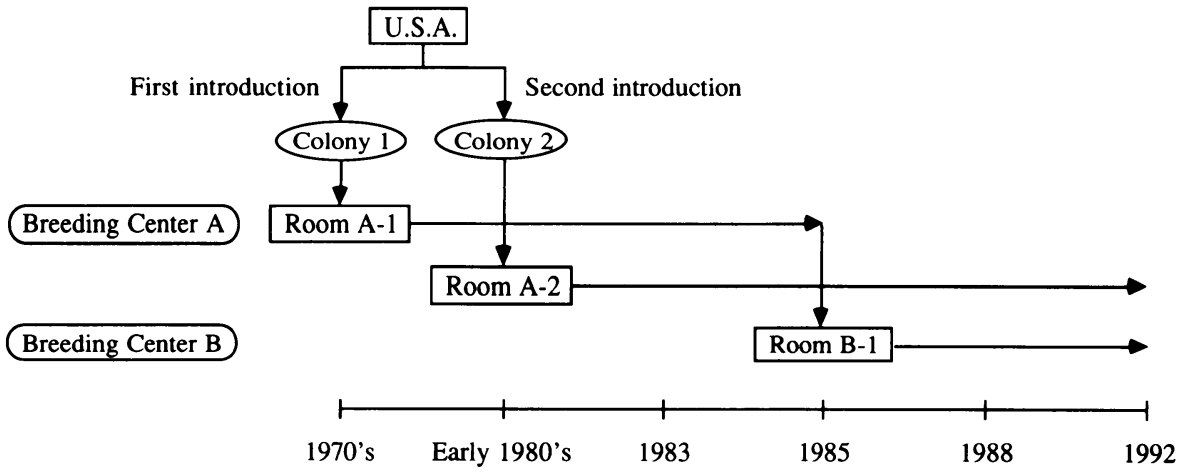

Fig. 1. History of colony introduction from the United States to Japan

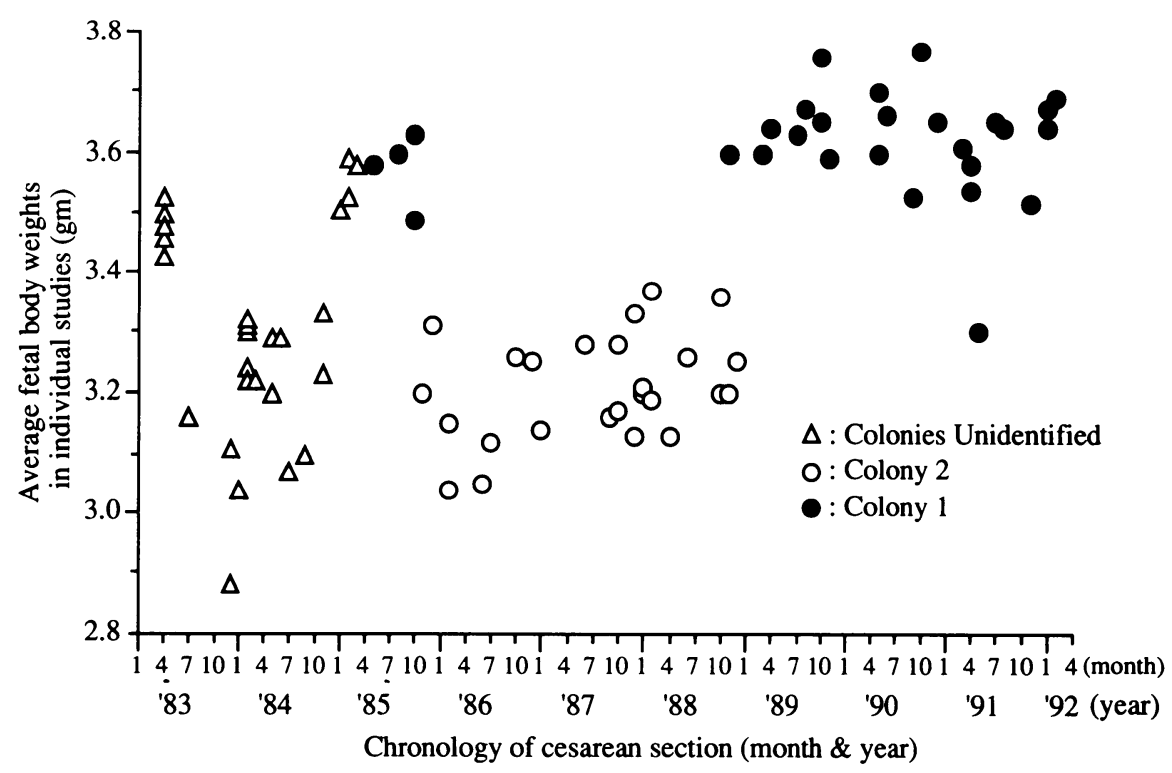

Fig. 2. Average fetal body weights in individual studies, showing the difference between colonies

Table 2. Comparison of reproductive status on gestational day 20 in Sprague-Dawley rats between Colonies 1, 2 and unidentified

\begin{tabular}{lccccc}
\hline Colonies & No. studies & No. implants & Resorption rate $(\%)$ & No. live fetuses & Fetal body weight $(\mathrm{g})$ \\
\hline Colony 1 & 29 & $15.0 \pm 0.9^{1}$ & $4.8 \pm 1.7$ & $14.3 \pm 0.9$ & $3.61 \pm 0.09$ \\
& & $(13.1-16.4)^{2}$ & $(1.9-8.7)$ & $(12.0-15.7)$ & $(3.30-3.77)$ \\
Colony 2 & 25 & $15.4 \pm 0.9$ & $5.7 \pm 2.0$ & $14.5 \pm 0.9$ & $3.21 \pm 0.09^{*}$ \\
& & $(12.6-16.6)$ & $(1.3-9.8)$ & $(11.7-15.7)$ & $(3.04-3.37)$ \\
& & & $*$ Significant difference $(\mathrm{P} \leq 0.05)$ between Colonies 1 and 2 \\
\hline Colony & 27 & $14.2 \pm 1.1$ & $5.9 \pm 1.6$ & $13.4 \pm 1.1$ & $3.30 \pm 0.18$ \\
unidentified & & $(12.0-15.9)$ & $(3.5-10.4)$ & $(11.5-15.4)$ & $(2.88-3.59)$ \\
\hline
\end{tabular}

$$
1: \text { Mean } \pm \text { S.D. } \quad 2: \text { Range }
$$




\section{$\mathrm{SD}$ 系ラットにおける胎児体重の変動に関する調查}

坂芳樹・小松哲郎・中塚敏夫・松本浩良

近藤最・藤井孝朗*

萬有製薬株式会社開発研究所

*萬有製薬株式会社研究開発本部

我々が過去 9 年間に亘って実施してきた SpragueDawley 系ラットを用いた生殖発生毒性試験81実験の 繁殖状況に関する対照データのうち, 着床数, 死肧率, 生存胎児数等のデータに関しては安定した值が得ら れてきたが, 妊娠20日秢の生存胎児体重值に関しての
み大きな変動がみられた。ブリーダーとの共同調査の 結果, この胎児体重値の变動は同系統ラットを米国よ

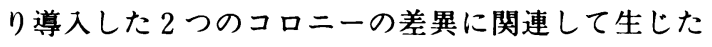
ことが判明した。 